\title{
Dor musculoesquelética em motoristas e cobradores de ônibus da Região Metropolitana de Belo Horizonte, Brasil
}

\author{
Musculoskeletal pain among bus drivers and fare collectors \\ in the Metropolitan Region of Belo Horizonte, Brazil
}

Mariana Roberta Lopes Simões ${ }^{1}$

Ada Ávila Assunção ${ }^{2}$

Adriane Mesquita de Medeiros ${ }^{2}$

${ }^{1}$ Programa de PósGraduação em Assistência Farmacêutica, Faculdade de Farmácia, UFRGS, Porto Alegre, RS, Brasil. marianarobertasimoes@ gmail.com

${ }^{2}$ Faculdade de Medicina, Universidade Federal de Minas Gerais. Belo Horizonte MG Brasil.

\begin{abstract}
Musculoskeletal pain among professionals in the transport sector has been linked to working conditions. The scope of this study was to assess the prevalence of cervical musculoskeletal pain and its relation to pain in other areas (arms, hands and shoulders). The association between neck pain, related to pain in other areas or otherwise, was checked against occupational factors. A cross-sectional, descriptive and analytical study was conducted with 799 bus drivers and 708 fare collectors of the Metropolitan Region of Belo Horizonte, Brazil. The outcome was characterized according to the positive answer to the question about musculoskeletal pain in the anatomical areas studied. The prevalence of neck pain in the sample was highest at 16.3\%, followed by pain in the shoulders $15.4 \%$, arms $13.3 \%$ and hands $6.3 \%$. The factors associated with musculoskeletal pain in the sample were being female, complaints of disability, perception of threat to safety, vibration, excessive or unbearable noise and sitting in an uncomfortable posture. The results provide clues to transformation of the workplace, thereby contributing to the enhancement of occupational health.
\end{abstract}

Key words Occupational health, Musculoskeletal pain, Working conditions, Transportation
Resumo A dor musculoesquelética em profissionais do setor de transporte tem sido relacionada às condições de trabalho. Objetivou-se estimar a prevalência de dor cervical e sua relação com a dor em outros sítios (braços, mãos e ombros). Verificou-se a associação entre dor no pescoço, relacionada ou não à dor nos outros sítios, com os fatores ocupacionais. Estudo transversal, descritivo e analítico. Foram entrevistados 799 motoristas e 708 cobradores de ônibus da Região Metropolitana de Belo Horizonte, Brasil. Para o desfecho foi considerada a resposta sobre a existência de dor por sítio anatômico. A prevalência de dor musculoesquelética no pescoço foi de 16,3\%. Para ombros, braços e mãos foram $15,4 \%, 13,3 \%$ e $6,3 \%$, respectivamente. Aqueles com dor no pescoço apresentaram maiores prevalências de dores nas demais áreas estudadas. Os fatores associados à dor musculoesquelética foram sexo feminino, relato de incapacidade, percepção de ameaça a segurança, vibração, ruído elevado ou insuportável e adoção de postura desconfortável. Os resultados indicaram a elaboração de pistas para transformação do ambiente de trabalho, de maneira a contribuir para a promoção da saúde dos trabalhadores.

Palavras-chave Saúde do trabalhador, Dor musculoesquelética, Condições de trabalho, Transportes 


\section{Introdução}

Fatores ocupacionais influenciam a precipitação e a evolução da dor musculoesquelética em trabalhadores ${ }^{1}$, levando a prejuízos na qualidade de vida do indivíduo ${ }^{2}$. Nos últimos anos, as pesquisas sobre os sintomas musculoesqueléticos têm sido realizadas no setor de serviços, por exemplo, o transporte urbano ${ }^{3}$, ultrapassando o foco anteriormente concentrado no setor industrial.

A dor musculoesquelética aumenta as chances de afastamento do trabalho ${ }^{4}$. Tal situação acarreta prejuízos para as empresas e para a sociedade em geral devido à queda da produtivida$\mathrm{de}^{2}$ associada à diminuição da capacidade laboral e aposentadorias precoces, como evidenciado nos Estados Unidos ${ }^{5}$ e nos países da União Européia ${ }^{6}$.

No Brasil, a magnitude do problema é pouco conhecida porque as fontes de dados nem sempre permitem desagregar informações sobre o assunto, ou então, como é o caso da previdência social, não abrangem o conjunto dos trabalhadores expostos ${ }^{7}$. Quanto a esta, as informações disponíveis sobre número e causas dos afastamentos referem-se à população segurada, que representa $50 \%$ da população economicamente ativa. Ainda assim, seguindo uma tendência crescente desde os anos anteriores, em 2014 foram concedidos 439.157 auxílios-doença por agravos musculoesqueléticos entre os segurados. Tal estatística ocupa o segundo lugar no ranking nacional de afastamentos do trabalho por doença no referido ano ${ }^{8}$.

Os estudos sobre dor musculoesquelética na população adulta em geral ${ }^{9} \mathrm{e}$ na população trabalhadora especificamente ${ }^{10,11}$ abordam frequentemente sintomas nas extremidades superiores, em particular a região cervical, a qual é considerada uma região anatômica vulnerável. A dor musculoesquelética localizada no pescoço é fonte de elevados gastos com assistência médica ${ }^{12}$, sendo referida como quarta causa de anos perdidos por incapacidade na população adulta9 .

Em motoristas e cobradores, a dor cervical assume destaque quando estudamos as dores musculoesqueléticas de acordo com a região do cor$\mathrm{po}^{13-16}$. Entre esses trabalhadores são evidenciadas prevalências de dor cervical que variam de $30 \mathrm{a}$ $50 \%{ }^{13,15-17}$. Tal sintoma está fortemente associado com os fatores ocupacionais ${ }^{10,12}$. No setor de transporte são conhecidos os seguintes fatores de risco: a postura estática, os movimentos repetitivos desencadeados de forma brusca e a vibração ${ }^{14,18}$. No grupo dos fatores psicossociais foram descritas associações com altas demandas de trabalho, baixo suporte e alta exigência psicológica ${ }^{13,16}$.
A dor musculoesquelética cervical ocorre isoladamente ou combinada com dor em outras regiões do corpo como membros superiores e ombros $^{11,14}$. Em epidemiologia, é recente o estudo da dor musculoesquelética referida em mais de um sítio anatômico ${ }^{19}$. Se em mais de um sítio, o quadro é considerado mais grave devido ao maior espectro de limitações e incapacidade que pode ocasionar para o indivíduo quando comparado ao quadro de dor localizada em um único sítio ${ }^{19,20}$. A dor difusa, em múltiplos sítios, impacta substancialmente a aptidão física, a saúde geral, as atividades da vida diária ${ }^{21}$ e do trabalho dos sujeitos acometidos. Apesar de comum na população ativa ${ }^{20}$, são escassos os estudos no tema ${ }^{13}$. No Brasil, além de raros estudos dedicados a examinar a situação dos rodoviários, não foram identificadas investigações cujo foco seja a relação da dor cervical com a dor em outros sítios corporais. Vale destacar o estudo de Fernandes et al. ${ }^{22}$, realizado no grupo de trabalhadores da indústria de plásticos que trouxe resultados esclarecedores sobre os distúrbios musculoesqueléticos de origem ocupacional.

O objetivo da presente pesquisa foi estimar a prevalência de dor musculoesquelética cervical e sua relação com a dor em outros sítios (braços, mãos e ombros) em motoristas e cobradores do transporte coletivo de ônibus da Região Metropolitana de Belo Horizonte (RMBH). Objetivou-se também verificar a associação entre dor no pescoço, relacionada ou não a dor em outros sítios, com os fatores ocupacionais.

\section{Metodologia}

Este inquérito epidemiológico foi desenvolvido com o apoio do Ministério Público do Trabalho de Minas Gerais e dos sindicatos das empresas e dos trabalhadores do transporte urbano. Tais atores, sensíveis às reivindicações dos trabalhadores, demandaram a compreensão da real condição de trabalho e saúde no setor por meio do método científico. A parceria favorece, através dos dados gerados pela pesquisa, o planejamento de ações de melhorias nos ônibus. A operacionalização do trabalho de campo foi iniciada após uma ampla divulgação da pesquisa e de seus objetivos pela Rádio Favela, além de folhetos e cartazes distribuídos nos locais de acesso dos trabalhadores foco do estudo.

A população elegível foi a de motoristas e cobradores das empresas de transporte coletivo urbano das cidades de Belo Horizonte, Betim e Contagem. Para a realização do estudo, foi se- 
lecionada uma amostra não probabilística, por conveniência, considerada em quotas proporcionais ao total dos profissionais de cada cidade e estratificadas por ocupação (motoristas e cobradores). Durante a coleta de dados, tomou-se como universo 17.470 trabalhadores. Para o cálculo amostral, foi considerada a prevalência de 50\% para o desfecho dor nas costas ${ }^{17}$, erro amostral de $4 \%$ e intervalo de confiança de $95 \%$. Com base nesses critérios, obteve-se a estimativa de 1.126 sujeitos (565 motoristas e 561 cobradores).

A coleta de dados face a face foi realizada por 22 entrevistadores treinados entre abril e junho de 2012. Para a coleta, foi desenvolvido questionário digital, composto por questões socioeconômicas, ocupacionais, de saúde e hábitos de vida. A coleta ocorreu nos períodos da manhã e da tarde, em quatro das cinco estações de ônibusmetrô de Belo Horizonte e em 35 dos 244 pontos de conforto das três cidades. O instrumento e os procedimentos do inquérito foram testados em uma etapa piloto $(\mathrm{n}=30)$. Os indivíduos que participaram do estudo piloto não foram incluídos na amostra final.A confiabilidade das respostas foi aferida pela reaplicação de perguntas a $12 \%$ dos participantes do inquérito.

A pesquisa foi aprovada pelo Comitê de Ética em Pesquisa da Universidade Federal de Minas Gerais e não possui conflito de interesse. $\mathrm{O}$ Termo de Consentimento Livre e Esclarecido foi assinado por todos os participantes do estudo.

As variáveis "dor", no pescoço, nos braços, nas mãos e nos ombros, foram aferidas separadamente, por meio de pergunta direta, com a seguinte estrutura: Abaixo estão listados alguns problemas de saúde. Se você não possui o problema, fale NUNCA. Se você sente o problema, fale com que frequência ele acontece. - Dor no pescoço e assim para todas as partes do corpo investigadas. Ao entrevistado, foram dadas as seguintes possibilidades de resposta à questão: nunca, raramente, pouco frequente, frequente e muito frequente. Foram consideradas positivas para dor as respostas frequente ou muito frequente por seguimento corporal, resultando em eventos binários (SIM e NÃO).

Tal estratégia metodológica de dimensionamento da dor musculoesquelética no momento da pesquisa é referida em estudos ocupacionais que abordam diferentes categorias de trabalhadores $^{23-25}$. Considera-se ainda que o autorrelato do estado de saúde seja uma ferramenta importante em saúde do trabalhador no Brasil, principalmente por serem raras outras fontes de informação seguras e disponíveis sobre o tema.
A metodologia que considera relatos de trabalhadores é comum nos estudos epidemiológicos por todo o mundo ${ }^{26}$. Sendo reconhecida no meio científico a contribuição de inquéritos no estudo especifico dos distúrbios musculoesqueléticos ${ }^{1}$.

As variáveis explicativas foram classificadas em quatro blocos, quais sejam:

- 1o Bloco - Sociodemográfico: sexo, idade, escolaridade, renda familiar e a cor autorreferida;

- 2o Bloco - Saúde e hábitos de vida: frequência semanal de atividade física, autoavaliação de saúde, relato de incapacidade pela dor nas atividades diárias e de trabalho nas últimas 4 semanas e índice de massa corporal;

- 3o Bloco - Psicossocial/Vitimização: segurança pessoal ameaçada no trabalho, episódio de agressão no trabalho nos últimos 12 meses e pensamento de mudança de local de trabalho devido a episódios de agressão ou ameaça;

- 4o Bloco - Ocupacional: percepção do ruído, da temperatura, da iluminação, da vibração e do trânsito, postura desconfortável e realização de dobras ou hora extras;

Foram considerados possíveis fatores de confusão o cargo (motorista ou cobrador) e o tempo no cargo.

Para a análise dos dados, primeiramente foi realizada uma abordagem descritiva da população amostrada. Em seguida, foi realizada uma análise bivariada, que testou a ocorrência simultânea de dor no pescoço com a dor em cada um dos outros seguimentos estudados (mãos, ombros e braços). No próximo passo, testou-se a associação da dor no pescoço com cada variável explicativa dos quatro blocos. Nessa etapa, foram selecionadas as variáveis associadas à dor no pescoço com $p \leq 0,20$. Tais variáveis foram inseridas uma a uma em modelos intermediários de análise múltipla, considerando apenas as pertencentes ao mesmo bloco. Finalmente, foram retidas em cada bloco as que mantiveram associação com a dor no pescoço ao nível de 5\%.

O modelo multivariado foi construído ajustando gradualmente as variáveis de um bloco às variáveis retidas no bloco anterior, na seguinte ordem: variáveis sociodemográficas; de saúde e hábitos de vida; psicossociais/vitimização; ocupacionais. Utilizou-se a estratégia de deleção sequencial das variáveis no modelo, permanecendo as variáveis associadas à dor no pescoço ao nível de $5 \%$.

A última etapa consistiu no teste da mesma modelagem descrita acima ajustada em dois modelos distintos: o primeiro, ajustado pelas variáveis cargo e anos no cargo; e o segundo, ajustado 
pelas variáveis dor nos ombros, dor nos braços e dor nas mãos.

Foi utilizada a Razão de Prevalência (RP) para aferir a magnitude das associações estatísticas entre o desfecho e as variáveis dos blocos analisados. As magnitudes de associação foram obtidas por regressão de Poisson, com variância robusta e intervalo de confiança de 95\% (IC95\%). Foi utilizado o pacote estatístico Stata, versão 12.1.

\section{Resultados}

Do universo de 17.470 trabalhadores do transporte coletivo urbano das cidades investigadas, participaram do inquérito 1.607 indivíduos. Destes, 1507 (799 motoristas e 708 cobradores) completaram a pergunta que originou o desfecho da presente investigação (perda de 6\%). Não houve diferença significativa entre as características sociodemográficas do grupo que respondeu e do que não respondeu à pergunta de interesse.

A amostra foi constituída predominantemente por homens (87\%), com idade inferior a 40 anos $(67,4 \%)$, média de 36,2 anos (DP $=10)$, nível médio de escolaridade $(64,5 \%)$, renda familiar entre 2 e 4 salários mínimos (49,7\%) e que se autodeclararam pardos ou negros $(73,4 \%)$. Considerando a saúde, $38,5 \%$ tinham sobrepeso (IMC entre 25,0 e 30,0 ) e $16 \%$, obesidade (IMC $\geq 30,0), 51,8 \%$ não praticavam atividade física e $27,2 \%$ relataram incapacidade nas atividades diárias por dor nas últimas 4 semanas. Dos respondentes, 79,8\% avaliaram sua situação de saúde como boa ou muito boa. No total, 53,1\% dos participantes eram motoristas e $56,1 \%$ com menos de 5 anos no cargo. Vibração, desconforto térmico e sonoro foram relatados por $39,3 \%$, $45 \%$ e $51,3 \%$ dos entrevistados, respectivamente. Predominaram os respondentes que consideraram a iluminação boa (67\%), o trânsito ruim $(84,5 \%)$ e que realizavam dobras ou hora extras $(44,8 \%)$. A postura desconfortável sempre ou quase sempre foi relatada por 43,1\%. Com relação à vitimização, 44,7\% relataram episódio de agressão nos últimos 12 meses, 53,6\% relataram ameaça no trabalho e $34,9 \%$ referiram pensamento de mudar de emprego devido à violência.

A prevalência de dor musculoesquelética no pescoço foi de $16,3 \%$, seguiu-se a prevalência de dor nos ombros $(15,4 \%)$, nos braços $(13,3 \%)$ e nas mãos (6,3\%). Dos 245 indivíduos com relatos de dor no pescoço, 66,9\% referiram também dor em pelo menos mais 1 dos outros 3 segmentos estudados. A Tabela 1 apresenta a ocorrência de dor musculoesquelética por região corporal de acordo com a presença ou ausência de dor no pescoço. Trabalhadores que relataram dor no pescoço apresentaram significativamente maiores prevalências de dor nos braços $(\mathrm{RP}=3,58$ IC95\%:2,88-4,43), nas mãos ( $\mathrm{RP}=3,81$ IC95\%: 3,02-4,80) e nos ombros ( $\mathrm{RP}=7,86 \mathrm{IC} 95 \%$ : 6,35$9,72)$.

A investigação dos fatores associados à dor foi iniciada por uma análise bivariada considerando a relação entre a dor no pescoço e as demais variáveis dos quatro blocos (Tabela 2). Na análise bivariada foram selecionadas as variáveis associadas ao desfecho ao nível de $\mathrm{p}<0,20$ que foram inseridas em uma análise intermediária, multivariada por bloco. $\mathrm{Na}$ análise intermediária foram identificadas as variáveis associadas à dor no pescoço com $\mathrm{p}<0,05$ para compor o modelo final. Foram elas: Bloco 1: sexo, escolaridade e renda familiar; Bloco 2: autoavaliação de saúde e incapacidade pela dor; Bloco 3: segurança pessoal ameaçada e pensamento de mudança de emprego por violência; Bloco 4: ruído, vibração e postura.

O modelo final sem ajustes apresentou como variáveis associadas à dor no pescoço o sexo feminino ( $\mathrm{RP}=1,45$; IC95\%:1,12-1,88); o relato de incapacidade nas atividades diárias de forma moderada ( $\mathrm{RP}=1,51$; IC95\%:1,13-2,02) e extrema $(\mathrm{RP}=2,21$; IC95\%:1,69-2,90); o relato de ameaça à segurança pessoal no trabalho

Tabela 1. Prevalência de dor musculoesquelética em motoristas e cobradores com e sem dor no pescoço. Belo Horizonte, Betim, Contagem, MG-2012.

\begin{tabular}{|c|c|c|c|c|c|c|c|}
\hline \multirow[t]{2}{*}{ Área da dor } & \multirow{2}{*}{$\begin{array}{c}\text { Prevalência } \\
\text { na amostra }\end{array}$} & \multicolumn{2}{|c|}{$\begin{array}{c}\text { Sem dor no } \\
\text { pescoço }(n=1262)\end{array}$} & \multicolumn{2}{|c|}{$\begin{array}{c}\text { Com dor no } \\
\text { pescoço }(n=245)\end{array}$} & \multirow[b]{2}{*}{ RP } & \multirow[b]{2}{*}{ IC 95\% } \\
\hline & & $\mathrm{N}$ & $\%$ & $\mathrm{~N}$ & $\%$ & & \\
\hline Braço & 13,4 & 114 & 9,0 & 87 & 35,5 & $3,58^{\star \star}$ & $2,88-4,43$ \\
\hline Mão & 6,3 & 45 & 3,6 & 50 & 20,4 & $3,81^{\star *}$ & $3,02-4,80$ \\
\hline Ombro & 15,4 & 87 & 6,9 & 144 & 58,8 & $7,86^{\star *}$ & $6,35-9,72$ \\
\hline
\end{tabular}

${ }^{*} \mathrm{n}=1507 \quad{ }^{* *} \mathrm{p}<0,001-$ Obtido pela Regressão de Poisson com variância robusta. 
Tabela 2. Análise bivariada entre dor no pescoço e características sociodemográficas, de saúde e hábitos de vida, psicossocial/vitimização e ocupacionais entre trabalhadores do transporte coletivo urbano da RMBH. Belo Horizonte, Betim e Contagem, MG - 2012.

\begin{tabular}{|c|c|c|c|}
\hline Variáveis & $\mathbf{n}^{\mathrm{a}}$ & Dor no pescoço \% & RP (IC95\%) \\
\hline \multicolumn{4}{|l|}{ Bloco 1 - Sociodemográficas } \\
\hline \multicolumn{4}{|l|}{ Sexo } \\
\hline Masculino & 1314 & 14,1 & 1 \\
\hline Feminino & 193 & 27,5 & $1,45(1,12-1,88)^{*}$ \\
\hline \multicolumn{4}{|l|}{ Idade (em anos) } \\
\hline $18-30$ & 524 & 17,2 & 1 \\
\hline $31-40$ & 495 & 14,3 & $0,83(0,62-1,11)$ \\
\hline $41-50$ & 345 & 17,4 & $1,01(0,75-1,36)$ \\
\hline $51-75$ & 143 & 16,8 & $0,67(0,65-1,47)$ \\
\hline \multicolumn{4}{|l|}{ Renda familiar (em salários mínimos) ${ }^{\mathrm{b}}$} \\
\hline Maior que 4 & 503 & 14,7 & 1 \\
\hline Entre 2 e 4 & 720 & 15,83 & $1,12(0,86-1,46)$ \\
\hline Menor que 2 & 225 & 23,1 & $1,64(1,20-2,25)^{*}$ \\
\hline \multicolumn{4}{|l|}{ Escolaridade } \\
\hline Superior/especialização (> 11 anos) & 49 & 27,9 & 1 \\
\hline Médio/técnico (9 a 11 anos) & 979 & 16,2 & $0,58(0,35-0,96))^{*}$ \\
\hline Fundamental ( $<9$ anos $)$ & 485 & 15,2 & $0,55(0,32-0,92)^{*}$ \\
\hline \multicolumn{4}{|l|}{ Cor da pele } \\
\hline Branca & 300 & 16,7 & 1 \\
\hline Parda/Negra & 1106 & 15,8 & $0,95(0,71-1,26)$ \\
\hline Indígena/Amarelo & 101 & 19,8 & $1,18 \mathrm{n}(0,74-1,89)$ \\
\hline \multicolumn{4}{|l|}{ Bloco 2 - Saúde e hábitos de vida } \\
\hline \multicolumn{4}{|l|}{ IMC } \\
\hline $18,0-24,9$ & 657 & 16,2 & 1 \\
\hline $25,0-29,9$ & 558 & 14,8 & $0,91(0,70-1,18)$ \\
\hline$\geq 30$ & 232 & 20,3 & $1,24(0,91-1,69)$ \\
\hline \multicolumn{4}{|c|}{$\begin{array}{l}\text { Influência da DOR nas últimas } 4 \text { semanas nas atividades } \\
\text { diárias e de trabalho }\end{array}$} \\
\hline De maneira nenhuma/pouco & 1096 & 11,1 & 1 \\
\hline Moderado & 246 & 22,4 & $2,08(1,50-2,67)^{\star}$ \\
\hline Bastante/Extremamente & 163 & 41,7 & $3,74(2,92-4,79)^{*}$ \\
\hline \multicolumn{4}{|l|}{ Autoavaliação de saúde } \\
\hline Boa/ muito boa & 1200 & 13,7 & 1 \\
\hline Regular & 280 & 25,0 & $1,81(1,41-2,32)^{\star}$ \\
\hline Ruim/ muito ruim & 24 & 41,1 & $3,03(1,84-4,96)^{*}$ \\
\hline \multicolumn{4}{|l|}{ Pratica de atividade física } \\
\hline 3 ou mais vezes na semana & 343 & 12,8 & 1 \\
\hline $1-2$ vezes na semana & 382 & 12,0 & $0,93(0,63-1,38)$ \\
\hline Nunca & 781 & 19,8 & $1,54(1,13-2,10)^{\star}$ \\
\hline
\end{tabular}


Tabela 2. continuação

\begin{tabular}{|c|c|c|c|}
\hline Variáveis & $\mathbf{n}^{\mathrm{a}}$ & Dor no pescoço \% & RP (IC95\%) \\
\hline \multicolumn{4}{|l|}{ Bloco 3 - Psicossocial/vitimização } \\
\hline \multicolumn{4}{|c|}{ Ameaça a segurança pessoal no trabalho } \\
\hline Não & 682 & 9,5 & 1 \\
\hline Sim & 790 & 22,4 & $2,35\left(1,80-3,06^{*}\right)$ \\
\hline \multicolumn{4}{|c|}{ Episódio de agressão nos últimos 12 meses } \\
\hline Não & 813 & 13,28 & 1 \\
\hline $\operatorname{Sim}$ & 659 & 20,3 & $1,53(1,21-1,92)^{\star}$ \\
\hline \multicolumn{4}{|l|}{ Pensamento de mudança de emprego } \\
\hline Não & 957 & 12,7 & 1 \\
\hline Sim & 513 & 23,2 & $1,81(1,44-2,28)^{\star}$ \\
\hline \multicolumn{4}{|l|}{ Bloco 4 - Ocupacional } \\
\hline \multicolumn{4}{|l|}{ Hora extra } \\
\hline Nunca & 191 & 17,8 & 1 \\
\hline Raramente/Às vezes & 644 & 14,7 & $0,82(0,57-1,18)^{\star}$ \\
\hline Quase sempre/sempre & 672 & 17,3 & $0,96(0,68-1,37)^{\star}$ \\
\hline \multicolumn{4}{|l|}{ Vibração } \\
\hline Nunca & 508 & 8,0 & 1 \\
\hline Raramente/Às vezes & 411 & 14,3 & $1,77(1,22-2,59)^{\star}$ \\
\hline Sempre/quase sempre & 587 & 24,7 & $3,06(2,21-4,23)^{\star}$ \\
\hline \multicolumn{4}{|l|}{ Temperatura } \\
\hline Tolerável/Incomoda Pouco & 826 & 11,6 & 1 \\
\hline Incomoda muito/Insuportável & 681 & 21,9 & $1,88(1,49-2,38)^{\star}$ \\
\hline \multicolumn{4}{|l|}{ Iluminação } \\
\hline Boa & 1004 & 13,3 & 1 \\
\hline Regular & 376 & 20,7 & $1,55(1,21-2,00)^{\star}$ \\
\hline Ruim/muito ruim & 127 & 25,9 & $1,94(1,39-2,71)^{*}$ \\
\hline \multicolumn{4}{|l|}{ Ruído } \\
\hline Desprezível/razoável & 732 & 9,2 & 1 \\
\hline Elevado & 456 & 21,3 & $2,29(1,72-3,05)^{\star}$ \\
\hline Insuportável & 318 & 24,9 & $2,67(1,98-3,59)^{\star}$ \\
\hline \multicolumn{4}{|l|}{ Postura desconfortável } \\
\hline Nunca & 262 & 6,1 & 1 \\
\hline Raramente/Às vezes & 596 & 10,2 & $1,67(0,98-2,85)^{*}$ \\
\hline Sempre/quase sempre & 649 & 25,9 & $4,23(2,59-6,94)^{\star}$ \\
\hline \multicolumn{4}{|l|}{ Trânsito } \\
\hline Bom/Regular & 236 & 9,3 & 1 \\
\hline Ruim/muito ruim & 1271 & 17,5 & $1,88(1,24-2,85)^{*}$ \\
\hline
\end{tabular}

a Total de cada grupo - para algumas variáveis, a soma pode ser diferente de 1507 em função de informação não declarada (missing). b Salário mínimo de referência em abril de $2012=\mathrm{R} \$ 306,19 .{ }^{\star} \mathrm{p}<0,20$.

$(\mathrm{RP}=1,53 ;$ IC95\%:1,17-2,01); a percepção da vibração sempre ou quase sempre $(\mathrm{RP}=1,64$; IC95\%:1,15-2,32), a percepção do ruído como elevado (RP = 1,61; IC95\%:1,21-2,15) ou insu- portável ( $\mathrm{RP}=1,67$; IC95\%:1,24-2,26) e a adoção de postura desconfortável sempre ou quase sempre (RP = 2,47; IC95\%:1,48-4,13).

Após ajuste pelo cargo e tempo, duração no 
cargo (modelo 1) os resultados não foram alterados. Já no modelo 2, ajustado pelo relato de dor musculoesquelética nas mãos, braços e ombros, permaneceram associados à dor no pescoço o relato de ameaça a segurança pessoal, a percepção do ruído como elevado ou insuportável e a adoção de postura desconfortável sempre ou quase sempre. Tais resultados são apresentados pela Tabela 3.

\section{Discussão}

A prevalência de dor no pescoço, na amostra estudada, foi associada às prevalências de dor nos braços, mãos e ombros. Tal resultado é consistente uma vez que a dor musculoesquelética em múltiplos sítios e é comum na população trabalhadora ${ }^{20}$. Ainda assim, há controvérsias na interpretação dos resultados empíricos. No âmbito da reumatologia, quadros multiálgicos crônicos, com ou sem lesões teciduais, são atribuídos a processos neuro-hormonais. Mecanismos de sensibilização central são mencionados por autores para explicar esse tipo de dor. Ou seja, a sensi-

Tabela 3. Análise multivariada dos fatores associados à prevalência de dor no pescoço nos motoristas e cobradores do transporte coletivo urbano da RMBH. Belo Horizonte, Betim, Contagem, MG - 2012.

\begin{tabular}{|c|c|c|c|c|}
\hline Variáveis & $\begin{array}{l}\text { Prevalência }(\%) \\
\quad n=1507\end{array}$ & $\begin{array}{l}\text { Modelo bruto } \\
\text { RP (IC 95\%) }\end{array}$ & $\begin{array}{c}\text { Modelo 1 } \\
\text { RP (IC 95\%) }\end{array}$ & $\begin{array}{c}\text { Modelo } 2 \\
\text { RP (IC 95\%) }\end{array}$ \\
\hline \multicolumn{5}{|l|}{ Sociodemográficas } \\
\hline \multicolumn{5}{|l|}{ Sexo } \\
\hline Masculino & 14,6 & 1 & 1 & 1 \\
\hline Feminino & 27,5 & $1,45(1,12-1,88)^{*}$ & $1,57(1,18-2,09)^{\star}$ & $1,23(0,96-1,56)$ \\
\hline \multicolumn{5}{|l|}{ Saúde e hábitos de vida } \\
\hline \multicolumn{5}{|l|}{ Incapacidade nas atividades } \\
\hline De maneira nenhuma/pouco & 11,1 & 1 & 1 & 1 \\
\hline Moderado & 22,4 & $1,51(1,13-2,02)^{*}$ & $1,47(1,10-1,96)^{*}$ & $1,27(0,98-1,66)$ \\
\hline Bastante/Extremamente & 41,7 & $2,31(1,69-2,90)^{* *}$ & $2,05(1,56-2,70)^{\star *}$ & $1,24(0,95-1,60)$ \\
\hline \multicolumn{5}{|l|}{ Psicossocial/Vitimização } \\
\hline \multicolumn{5}{|l|}{$\begin{array}{l}\text { Ameaça a segurança pessoal } \\
\text { no trabalho }\end{array}$} \\
\hline Não & 9,5 & 1 & 1 & 1 \\
\hline Sim & 22,4 & $1,53(1,17-2,01)^{\star}$ & $1,60(1,22-2,09)^{\star *}$ & $1,47(1,15-1,88)^{\star}$ \\
\hline \multicolumn{5}{|l|}{ Ocupacionais } \\
\hline \multicolumn{5}{|l|}{ Vibração } \\
\hline Nunca & 8,1 & 1 & 1 & 1 \\
\hline Raramente/às vezes & 14,4 & $1,21(0,82-1,78)$ & $1,23(0,83-1,81)$ & $1,09(0,77-1,55)$ \\
\hline Sempre/quase sempre & 24,7 & $1,64(1,15-2,32)^{\star}$ & $1,70(1,21-2,41)^{\star}$ & $1,30(0,95-1,79)$ \\
\hline \multicolumn{5}{|l|}{ Ruído } \\
\hline Desprezível/razoável & 9,3 & 1 & 1 & 1 \\
\hline Elevado & 21,3 & $1,61(1,21-2,15)^{* *}$ & $1,60(1,20-2,13)$ & $1,49(1,15-1,92)^{*}$ \\
\hline Insuportável & 24,8 & $1,67(1,24-2,26)^{\star *}$ & $1,67(1,24-2,25)^{\star \star}$ & $1,57(1,19-2,07)^{* *}$ \\
\hline \multicolumn{5}{|l|}{ Postura desconfortável } \\
\hline Nunca & 6,1 & 1 & 1 & 1 \\
\hline Raramente/ás vezes & 10,2 & $1,56(0,92-2,65)$ & $1,66(0,97-2,83)$ & $1,52(0,95-2,44)$ \\
\hline Sempre/quase sempre & 25,9 & $2,48(1,48-4,13)^{\star *}$ & $2,58(1,54-4,31)^{\star *}$ & $2,17(1,38-3,41)^{* *}$ \\
\hline
\end{tabular}

RP: Razão de Prevalência. IC 95\%: intervalo de 95\% de confiança; ${ }^{*} \mathrm{p}<0,05 ;{ }^{* *} \mathrm{p}<0,01$; Modelo 1 ajustado por cargo e anos no cargo, Modelo 2 ajustado por dores nos braços, mãos e ombros, Teste de Hosmer\&Lemeshow $>0,05$. 
bilização de neurônios centrais aumentaria a resposta à dor, desencadeando estados de hiperalgesia em um ou em vários segmentos corporais ${ }^{27}$. Contudo, tratando-se de grupos ocupacionais, as evidências conclamam argumentos interpretativos de outra natureza. Voltaremos a esse ponto.

$\mathrm{Na}$ amostra estudada, $66,9 \%$ dos entrevistados com dor no pescoço relataram dor em outro segmento (ombro, mão ou braço). Motoristas e cobradores que relataram dor no pescoço apresentaram significativamente maiores prevalências de dor nos braços, nas mãos e nos ombros comparados aos que não relataram. Esse resultado também foi evidenciado entre motoristas israelenses ${ }^{14}$. Tal associação sugere medidas de proteção mais abrangentes, de maneira a abarcar fatores identificados como risco para os problemas musculoesqueléticos em diferentes sítios anatômicos.

Processos de dor relacionados à atividade do pescoço, ombro ou membros superiores, potencialmente podem refletir nos demais ${ }^{11}$. A estabilidade da coluna cervical é obtida graças à interação coordenada de diferentes estruturas neuromusculares. A posição do ombro, por exemplo, pode aumentar substancialmente os níveis de ativação neuromuscular necessários para manter tal estabilidade. Do mesmo modo, músculos do pescoço participam da estabilização dos ombros durante os movimentos ${ }^{28}$.

Os mecanismos funcionais de reorganização da atividade muscular e as alterações no padrão de ativação muscular podem levar à fadiga e à dor ${ }^{29}$. Ademais, um mesmo fator pode ser relacionado à dor musculoesquelética em diferentes sítios anatômicos ${ }^{11,19}$. A sobreposição de riscos individuais e ocupacionais é comumente citada para explicar os quadros multiálgicos em trabalhadores ${ }^{1,30}$.

Recentemente, os epidemiologistas têm se interessado em estudar a dor difusa em trabalhadores $^{19}$. Entretanto, são menos recentes as evidências de pior prognóstico quando são múltiplos os sítios de dor musculoesquelética: são mais graves e incapacitantes se comparados à dor em um único segmento anatômico ${ }^{20,21}$.

A prevalência de dor no pescoço evidenciada no presente estudo (16,3\%) foi menor se comparada aos resultados obtidos em outras amostras de motoristas e cobradores no Brasil, na Venezuela e na Itália, sendo respectivamente $36,6 \%{ }^{17}$, $43 \%{ }^{15}$ e $43,4 \%{ }^{16}$. Em relação à dor no ombro, a prevalência de $15,4 \%$ foi aproximada aos 14,7\% encontrada entre motoristas israelenses ${ }^{14}$, porém, menor que os $27 \%$ e $26 \%$ entre motoristas brasi- $\operatorname{leiros}^{17}$ e venezuelanos ${ }^{15}$, respectivamente. A prevalência de dor no braço foi $13,3 \%$, maior que a evidenciada entre motoristas na Coreia $(6,2 \%)^{3}$. Já a prevalência de dor nas mãos $(6,3 \%)$ foi comparável aos 6\% e 6,2\% encontrados na Venezue$\mathrm{la}^{15}$ e na Coreia ${ }^{3}$ respectivamente.

Possivelmente, as diferenças encontradas estão associadas à definição temporal utilizada em cada inquérito. No presente estudo, interrogouse sobre a dor no momento da pesquisa, enquanto os pesquisadores da Venezuela, da Itália e da Coreia perguntaram sobre a dor nos últimos 12 meses.

Apesar da falta de consenso metodológico, os resultados das diferentes pesquisas ${ }^{3,14-16}$ identificam a região cervical como a área corporal mais vulnerável ao adoecimento musculoesquelético, haja vista as maiores prevalências descritas se comparadas aos braços, aos ombros e às mãos.

As evidências de maior vulnerabilidade da região cervical em amostras de trabalhadores do setor do transporte coletivo podem ser devido aos diferenciais de exposição à carga biomecânica e aos fatores psicossociais específicos a que se expõem esses trabalhadores, com desvantagem para o referido grupo ${ }^{30,31}$. Em relação às cargas biomecânicas, destacam-se a postura sentada e os movimentos constantes da cabeça e do tronco ${ }^{14}$, os quais geram pressões localizadas nas estruturas musculoesqueléticas com reflexos para os grupamentos musculares na região do ombro e pescoço $^{32}$. Apoio para a região cervical é um aparato recomendado para aliviar as tensões musculares sobre os discos intervertebrais ${ }^{14}$, mas a concepção da cadeira dos motoristas e cobradores nem sempre segue essa recomendação ergonômica ${ }^{3}$.

Tratando-se da análise dos fatores associados à dor no pescoço, não foram evidenciadas diferenças entre a análise bruta e ajustada por cargo. Tal resultado permite concluir que os motoristas e cobradores estudados estão igualmente suscetíveis à morbidade musculoesquelética.

Nos modelos brutos e ajustados por cargo, evidenciou-se significativa diferença na prevalência de dor cervical entre os sexos. De modo geral, são bem documentados os efeitos dos diferenciais de gênero ${ }^{33}$ quando se analisa a prevalência de dor musculoesquelética ${ }^{20,34}$. No modelo ajustado pela variável dor nas demais regiões, mãos, braços e ombros, desaparece a diferença entre gêneros. Pesquisas identificam maior prevalência de dor em múltiplos sítios no grupo das mulheres quando comparado com o grupo dos homens ${ }^{19,20}$. Este resultado sugere que os diferenciais de gênero se expressam a depender da exten- 
são da dor, com desvantagem para as mulheres.

Vibração não ficou associada à dor no pescoço após ajuste para dores nas outras regiões investigadas. Tendo sido evidenciada a associação da vibração com a dor cervical nos modelos bruto e ajustado por cargo. É plausível supor que a exposição à vibração em rodoviários esteja associada à vigência de dores em mais de uma região corporal. Sabe-se que trabalhadores do transporte estão especialmente expostos à vibração de corpo inteiro ${ }^{13,18}$, cujo efeito é potencializado pelo estado de conservação das vias ${ }^{18}$ com destaque para os impulsos vibratórios gerados pelos movimentos frequentes de parada e arrancada dos ônibus ${ }^{18,26}$. É possível que a exposição à vibração desse tipo afete simultaneamente as estruturas da coluna vertebral e da região do pescoço e do ombro $^{16,20}$. Entretanto, os resultados das pesquisas ainda são controversos ${ }^{16,11}$. Em suma, a hipótese de relação entre a vibração e a dor musculoesquelética carece de maiores esclarecimentos ${ }^{16,34}$.

A associação da dor no pescoço com a variável incapacidade evidenciada no modelo bruto perdeu significância após ajuste por outras variáveis relacionadas à dor, tal como o ocorrido na análise de gênero e vibração. Os relatos de interferência da dor durante as atividades nas últimas quatro semanas podem ser reflexo da restrição ao desenvolvimento do trabalho, principalmente para aqueles com maior extensão do quadro álgico. O adoecimento musculoesquelético, de natureza insidiosa, inicialmente se apresenta com sintomas leves ou moderados, o que geralmente não impede a realização das atividades cotidianas nem das tarefas no trabalho ${ }^{26}$. Todavia, é recomendado reconhecer e interpretar os sinais iniciais de comprometimento da capacidade musculoesquelética entre os trabalhadores para intervir de maneira a evitar o provável agravamento do quadro ${ }^{2}$.

Evidenciou-se associação de relatos de postura desconfortável, sempre ou quase sempre, à dor no pescoço nos modelos bruto e ajustados. $\mathrm{Na}$ Coreia, observaram-se, em motoristas de ônibus, efeitos potentes da postura sobre a ativação muscular. Isto é, a atividade de dirigir é um importante fator de sobrecarga postural. Insuficiências na educação postural para conduzir os ônibus e deficiências ergonômicas ${ }^{3}$ são fatores explicativos. O conforto do motorista tem relação direta com a funcionalidade e o design dos ônibus.

$\mathrm{Na}$ avaliação do conforto, não basta identificar os problemas nas engrenagens e no motor dos ônibus ${ }^{35}$. Além do design do assento, a duração da posição sentada, entre outros aspectos, é consi- derada como fator de risco ${ }^{14,35}$. Posições extremas do pescoço foram adotadas durante um terço do tempo ao volante, sendo identificado um tipo de torção acentuada mesmo durante as paradas do ônibus ${ }^{36}$. As queixas de desconforto postural são proporcionais à duração da posição sentada. A postura sentada, entre outros prejuízos, modifica a curvatura natural da coluna e aumenta a pressão sobre a região posterior dos discos intervertebrais ${ }^{35}$. No Reino Unido, cerca de $14 \%$ de todos os casos de dor no pescoço e nos membros superiores foram atribuídos à flexão prolongada do pescoço ${ }^{37}$. Tal raciocínio pode ser aplicado na interpretação dos resultados encontrados no grupo dos cobradores, pois eles se mantêm por longos períodos na posição sentada, sem apoio adequado, além de adotarem torção de tronco e pescoço durante o desenvolvimento das tarefas.

A exposição ao ruído é comum entre trabalhadores do trânsito urbano ${ }^{15}$. Sabe-se dos efeitos de tal agente sobre a saúde humana, tanto auditivos quanto não auditivos ${ }^{38}$. Na presente pesquisa, a percepção do ruído elevado ou insuportável aumentou em aproximadamente $60 \%$ a probabilidade de dor no pescoço nos dois grupos de trabalhadores (Modelo bruto). Evidências sobre os efeitos não auditivos da exposição ambiental ao ruído têm aumentado, sugerindo tratar-se de um problema de saúde pública. Irritação, fadiga e distúrbios do sono são efeitos conhecidos ${ }^{38}$. Tais agravos potencializam o surgimento ou o agravamento do estresse nos ambientes de trabalho. A exposição ao estresse tem sido associada a uma série de alterações fisiológicas que interferem na atividade hormonal e regulatória, de modo a facilitar a transmissão da dor e diminuir os seus limiares ${ }^{19}$. Efeitos do estresse possivelmente alteram os períodos de ativação muscular e provocam aumento da tensão, levando à fadiga e a outros sintomas no pescoço e nos ombros ${ }^{39}$.

A prevalência de dor no pescoço foi maior entre aqueles que relataram ameaça à segurança pessoal no trabalho. É plausível que as situações cada vez mais frequentes de insegurança no setor de transporte ${ }^{40-42}$, ao gerarem medo e ansiedade, sobrecarreguem os trabalhadores. Suspeita-se que a sobrecarga mental aumente a tensão muscular porque mobilizam unidades motoras de baixo limiar, explicando a fadiga e, por conseguinte, os processos álgicos cervicais ${ }^{39}$. $\mathrm{O}$ ambiente psicossocial adverso, perigoso ou gerador de sofrimento e de insatisfação foi significativamente associado a dor no pescoço entre motoristas na Itália ${ }^{16}$. A vivência de ato violento ou ameaça no trabalho associou-se tanto ao nú- 
mero de doenças crônicas diagnosticadas quanto aos transtornos mentais na amostra estudada, de acordo com resultados publicados anteriormente ${ }^{41,43}$. Em Maputo, a exposição de motoristas e cobradores do transporte urbano à violência foi associada a uma significativa redução nos indicadores de qualidade de vida, em seus domínios social, psicossocial e, sobretudo, físico ${ }^{42}$. Além da exposição à hostilidade das ruas, as múltiplas demandas sobre as quais o trabalhador do transporte coletivo tem pouco controle ${ }^{40}$ possivelmente aumentam a sobrecarrega e a insatisfação. Nesse caso, são agravadas as dores cervicais relacionadas aos fatores biomecânicos ${ }^{31}$.

A presente pesquisa, apesar de abordar uma amostra não probabilística, atingiu número satisfatório de respostas válidas para o desfecho: $36 \%$ superior ao proposto na fase de elaboração do projeto. Ademais, considerando as asperezas do campo, é inédito, no Brasil, um estudo com tamanha amostra entrevistada no local de trabalho. Com relação ao possível erro de informação, ressalta-se que a confiabilidade das respostas foi aferida por meio da reaplicação de perguntas a $12 \%$ dos participantes.

A despeito da consistência dos resultados, os limites devem ser esclarecidos, em especial aqueles relacionados ao desenho transversal, tais como a temporalidade e o viés de sobrevivência ou do trabalhador sadio. Este último se relaciona ao fato de que, nos ambientes laborais, já não se encontram presentes aqueles indivíduos que adoeceram ou cujo adoecimento implicou em incapacidade, explicando a subestimação dos resultados relacionados à morbidade ${ }^{44}$. Quanto à temporalidade, considera-se a impossibilidade de concluir causalidade, uma vez que o desfecho e as variáveis explicativas são abordados ao mesmo tempo.

É importante salientar o caráter preventivo dos agravos ao sistema musculoesquelético. $\mathrm{Na}$ perspectiva da saúde pública, os resultados apresentados constituem um direcionamento para a elaboração de programas e ações em prol da melhoria dos serviços de transporte e da promoção da saúde dos trabalhadores. Nesse sentido a implantação de postos de trabalho ergonômicos e investimentos em manutenção de veículos desempenham papel importante para redução dos níveis de vibração ${ }^{18}$ e ruído. Em relação a esses fatores de risco, a norma no 15.570 , da Associação Brasileira de Normas Técnicas (2011) recomenda a aquisição de ônibus com motores traseiros e sistemas de direção equipados com assistência hidráulica ou elétrica como meio de diminuir a exposição dos motoristas e cobradores ${ }^{45}$.

A fim de melhorar a saúde dos trabalhadores do serviço de transporte público, os resultados da presente pesquisa sugerem necessidade de ações de segurança pública. Nesse aspecto, recomendase acompanhamento psicológico para fortalecer os sujeitos para o enfrentamento e negociação de conflitos no ambiente de trabalho ${ }^{46}$. Além disso, diminuir a superlotação é fundamental para minimizar conflitos entre usuários e trabalhadores. A instalação de câmeras de segurança e dispositivos de rastreio dos ônibus pode desencorajar os comportamentos agressivos e identificar os autores no caso de episódios violentos ${ }^{47}$.

Por fim, resultados como os apresentados serão úteis para transformar as situações dos trabalhadores reconhecidamente expostos a múltiplos riscos. No Brasil, em especial em Minas Gerais, há falhas no cumprimento das normas dos órgãos regulatórios. A insuficiência de investimentos em infraestrutura, em frota para atendimento da crescente demanda e em salários, constatada pelos órgãos públicos ${ }^{48}$ é determinante para a análise da saúde dos trabalhadores ${ }^{49}$. Tal cenário apresenta potenciais efeitos sobre a saúde e o desempenho dos sujeitos que estarão menos preparados para lidar com as demandas do trânsito e dos passageiros.

As prevalências de dor nos ombros, braços, mãos e pescoço encontradas entre motoristas e cobradores na presente pesquisa confirmam a exposição destes aos riscos ocupacionais. Quando analisados os fatores associados ao desfecho dor cervical, os resultados reforçam a influência dos fatores ambientais e biomecânicos (vibração, postura e ruído) suficientemente descritos na literatura específica. Além disso, sugerem relação importante da dor com a violência referida por meio da resposta positiva ao sentimento de ameaça no trabalho. No conjunto, os resultados corroboram a complexidade da dor musculoesquelética e constituem importante direcionamento para planejamento de estratégias de promoção da saúde no setor de transporte. 


\section{Colaboradores}

MRL Simões realizou as análises estatísticas, estruturou o artigo, revisou a literatura, elaborou a primeira versão e colaborou na revisão final, aprovando-a. AA Assunção delineou e coordenou a pesquisa, colaborou na redação do manuscrito e na revisão final, aprovando-o. AM Medeiros colaborou na análise dos dados e na revisão do manuscrito.

\section{Referências}

1. Punnett L. Musculoskeletal disorders and occupational exposures: How should we judge the evidence concerning the causal association? Scand J Public Health 2014; 42(Supl. 13):49-58.

2. Hagberg M, Violante FS, Bonfiglioli R, Descatha A, Gold J, Evanoff B, Sluiter JK. Prevention of musculoskeletal disorders in workers: classification and health surveillance - statements of the scientific.Commitee on Musculoskeletal disorders of the International Commission on Occupational Health. BMC Musculoskeletal Disorders 2012; 13:109.

3. Lee JH, Gak HB. Effects of self stretching on pain and musculoskeletal symptom of bus drivers. J Phys Ther Sci 2014; 26(12):1911-1914.

4. Sérazin $\mathrm{C}, \mathrm{Ha} \mathrm{C}$, Bodin J, Imbernon E, Roquelaure Y. Employment and occupational outcomes of workers with musculoskeletal pain in a French region. Occup. Environ. Med. 2013; 70(3):143-148.

5. Bhattacharya A. Costs of occupational musculoskeletal disorders (MSDs) in the United States. International J Industrial Ergonomics 2014; 44(3):448-454.

6. European Agency for Safety and Health at Work (EU-OSHA). OSH in Figures: Work-Related Musculoskeletal Disorders in the EU - Facts and Figures. Luxembourg: Publications Office of the European Union; 2010.

7. Barbosa-Branco A, Souza WR, Steenstra A. Incidence of work and non-work related disability claims in Brazil. Am J Ind Med 2011; 54(11):858-871.

8. Brasil. Ministério da Previdência Social (MPS). Anuário Estatístico da previdência social. Brasília: MPS; 2013.

9. Hoy D, March L, Brooks P, Blyth F, Woolf A, Bain C, Smith E, Vos T, Barendregt J, Blore J, Murray, C, Burstein R, Buchbinder R. The global burden of neck pain: estimates from the Global Burden of Disease 2010 study. Ann Rheum Dis 2014; 73(7):1309-1315.

10. Mclean SM, May S, Klaber-Moffett J, Sharp DM, Gardiner E. Risk factors for the onset of non-specific neck pain: a systematic review. J Epidemiol Community Health 2010; 64(7):565-552.

\section{Agradecimentos}

Ao Ministério da Ciência, Tecnologia e Inovação - Conselho Nacional de Desenvolvimento Científico e Tecnológico.
11. Petit A, Ha C, Bodin J, Parot-Schinkel E, Ramond A, Leclerc A,Imbernon E, Roquelaure Y. Personal, Biomechanical, Organizational and Psychosocial Risk Factors for Neck Disorders in a Working Population. J of occup health 2014; 56(2):134-140.

12. Yang H, Haldeman S, Nakata A, Choi B, Delp L, Baker D. Work-Related Risk Factors for Neck Pain in the US Working Population. Spine 2015; 40(3):184-192.

13. Tamrin SBM, Yokoyama K, Aziz N, Maeda S. Association of risk factors with musculoskeletal disorders among male commercial bus drivers in Malaysia. Human Factors and Ergonomics in Manufacturing \& Service Industries 2014; 24(4):369-385.

14. Alperovitch-Najenson D. Katz-leurer M, Santo Y, Golman D, Kalichman L. Upper body quadrant pain in Bus Drivers. Arch Environ Occup Health 2010; 65(4):218223.

15. Fernandez-D’Pool J, Vélez A, Brito A, D’Pool C. Síntomas musculoesqueléticos en conductores de buses de una institución universitaria. Invest Clin 2012; 53(2):125-137.

16. Bovenzi M. A prospective Cohort Study of Neck and Shoulder Pain in Professional Drivers. Ergonomics 2015; 58(7):1-14.

17. Moura Neto AB, Silva MC. Diagnóstico das condições de trabalho, saúde e indicadores do estilo de vida de trabalhadores do transporte coletivo da cidade de Pelotas- RS. Rev Bras Ativ Fis Saúde 2012; 17(5):347-358.

18. Lewis CA, Johnson PW. Whole-body vibration exposure in metropolitan bus drivers. Occup Med 2012; 62(7):519-524.

19. Neupane S, Miranda H, Virtanen P, Siukola A, Nygard $\mathrm{CH}$. Do physical or psychosocial factors at work predict multi-site musculoskeletal pain? A 4-year follow-up study in an industrial population. Int Arch Occup Environ Health 2013; 86(5):581-589.

20. Herin F, Vézina M, Thaon I, Soulat JM, Paris C. Predictive risk factors for chronic regional and multisite musculoskeletal pain: A 5-year prospective study in a working population. Pain 2014; 155(5):937-943. 
21. Kamaleri Y, Natvig B, Ihlebaek CM, Benth JS, Bruusgaard D. Change in the number of musculoskeletal pain sites: A 14-year prospective study. Pain 2009; 141(1):25-30.

22. Fernandes RCP, Assunção AA, Silvany Neto AM, Carvalho FM. Musculoskeletal disorders among workers in plastic manufacturing plants. Rev Bras Epidemiol 2010; 13(1):11-20.

23. Steinmetz A, Scheffer I, Esmer E, Delank KS, Peroz I. Frequency, severity and predictors of playing-related musculoskeletal pain in professional orchestral musicians in Germany. Clin Rheumatol 2015; 34(5):965-973.

24. Pensri P, Janwantanakul P, Worakul P, Sinsongsook T. Biopsychosocial factors and perceived disability in saleswomen with concurrent low back pain. Saf Health Work 2010; 1(2):149-157.

25. Rodríguez-Romero B, Pita-Fernández S, Carballo-Costa L. Impact of physical and psychosocial factors on disability caused by lumbar pin amongst fishing sector workers. Rheumatol Int 2013; 33(7):1769-1778.

26. Szetto GPY, Lam P. Work-related musculoskeletal disorders in urban bus drivers of Hong Kong. J. Occup. Rehabil 2007; 17(2):181-198.

27. Curatolo M, Arendt-Nielsen L. Central hypersensitivity in chronic musculoskeletal pain. Phys Med Rehabil Clin NAm 2015; 26(2):175-184.

28. Nimbarte AD, Zreiqat $M$, Ning X. Impact of shoulder position and fatigue on the flexion-relaxation response in cervical spine. Clinical Biomechanics 2014; 29(3):277-282.

29. Madeleine P. On functional motor adaptations: from the quantification of motor strategies to the prevention of musculoskeletal disorders in the neck--shoulder region. Acta Physiologica 2010; 199(Supl. 679):1-46.

30. Schulte PA, Pandalai S,Wulsin V Chun H. Interaction of occupational and personal risk factors in workforce health and safety. Am J Public health 2012; 102(3):434448.

31. Widanarko B, Legg S, Devereux J, Stevenson M. The combined effect of physical, psychosocial/organisational and/or environmental risk factors on the presence of work-related musculoskeletal symptoms and its consequences. Appl Ergon 2014; 45(6):1610-1621.

32. Nordander C, Hansson GA, Ohlsson K, Arvidsson I, Balogh I, Stromberg U, Rittner R, Skerfving S. Exposure-response relationships for work-related neck and shoulder musculoskeletal disorders-Analyses of pooled uniform data sets. Appl Ergon 2016; 55:70-84.

33. Hirata $H$, Kergoat D. Novas configurações da divisão sexual do trabalho. Cadernos de Pesquisa. Fundação Carlos Chagas 2007; 37(132):595-609.

34. Larsson B, Sogaard K, Rosendal L. Work related neck-shoulder pain: a review on magnitude, risk factors, biochemical characteristics, clinical picture and preventive interventions. Best Pract Res Clin Rheumatol 2007; 21(3):447-463.

35. Smith J, Mansfield N, Gyi D, Pagett M, Bateman B. Driving performance and driver discomfort in an elevated and standard driving position during a driving simulation. Appl Ergon 2015; 49:25-33.

36. Albert WJ, Everson D, Rae M, Callaghan JP, Coll J, Kuruganti $U$. Biomechanical and ergonomic assessment of urban transit operators. Work 2014; 47(1):33-44.
37. Sim J, Lacey RJ, Lewis M. The impact of workplace risk factors on the occurrence of neck and upper limb pain: a general population study. BMC Public Health 2006; 6:234-244.

38. Portela BS, Queiroga RM, Constantini A, Sannin HTP. Annoyance evaluation and the effect of noise on the health of bus drivers. Noise health 2013; 15(66):301306.

39. Magnavita N., Elovainio M, Nardis I, Heoniemi T, Bergamaschi A. Environmental discomfort and musculoskeletal disorders. Occup Med 2011; 61(3):196-201.

40. Johansson G, Evans GW, Cederströn C, Rydstedt LW, Fulller-Rowell T, Ong AD. The effects of urban bus driving on blood pressure and musculoskeletal problems: quasi-experimental study. Phychosom Med 2012; 74(1):89-92.

41. Assunção AA, Medeiros AM. Violência a motoristas e cobradores de ônibus metropolitanos, Brasil. Rev Saude Publica 2015; 49(11):1-10

42. Couto MT. Workplace violence nature, prevalence and risk factors among drivers and conductors in Maputo city, Mozambique. Injury prevention 2010; 16(Supl. 1):139.

43. Assunção AA, Silva LS. Condições de trabalho nos ônibus e os transtornos mentais comuns em motoristas e cobradores. Região Metropolitana de Belo Horizonte. Minas Gerais, Brasil, 2012. Cad Saude Publica 2013; 29(12):2473-2486.

44. Shah D. Healthy worker effect phenomenon. Indian J Occup Environ Med 2009; 13(2):77-79.

45. Associação Brasileira de Normas Técnicas (ABNT). ABNT NBR 15570:2011: transporte - especificações técnicas para fabricação de veículos de características urbanas para transporte coletivo de passageiros. São Paulo: ABNT; 2011.

46. Puy J, Romain-Glassey N, Gut M, Pascal W, Mangin P, Danuser B. Clinically assessed consequences of workplace physical violence. Int Arch Occup Environ health 2015; 88(2):213-224.

47. Natarajan M, Clarke R, Carcach C, Ponce CS. Situational prevention and public transport crime in El Salvador. Crime Science 2015; 4(1):1-15.

48. Empresa de Transporte e Trânsito de Belo Horizonte (BHTRANS S/A). Elementos de Padronização Externa. Belo Horizonte: BHTRANS; 2011.

49. Chung Y-S, Wong J-T. Developing effective professional bus driver health programs: An investigation of self-rated health. Accident Analysis Prevention 2011; 43(6):2093-2103.

Artigo apresentado em 18/05/2016

Aprovado em 22/07/2016

Versão final apresentada em 24/07/2016 